\title{
2018 Status of the Measurement Capabilities for Fusion Gyrotrons at KIT/IHM
}

\author{
T. Ruess ${ }^{1}$, K. A. Avramidis ${ }^{1}$, M. Fuchs ${ }^{1}$, G. Gantenbein ${ }^{1}$, S. Illy ${ }^{1}$, Z. Ioannidis ${ }^{1}$, F. C. Lutz ${ }^{1}$, \\ S. Ruess ${ }^{1,2}$, T. Rzesnicki ${ }^{1}$, M. Thumm ${ }^{1,2}$, D. Wagner ${ }^{3}$, J. Weggen ${ }^{1}$ and J. Jelonnek ${ }^{1,2}$ \\ ${ }^{1}$ IHM, ${ }^{2}$ IHM Karlsruhe Institute of Technology (KIT), Kaiserstr. 12, D-76131 Karlsruhe, Germany, tobias.ruess@kit.edu \\ ${ }^{3}$ Max Planck Institute for Plasma Physics, Boltzmannstr. 2, D-85748 Garching, Germany
}

\begin{abstract}
This paper gives an overview about three key measurement capabilities at KIT/IHM for fusion gyrotrons. The discussed topics are: RF window test facility, low power launcher test setup and the frequency measurement setup placed at the high power test facility. Further, approaches for upgrading the setups to frequencies above $200 \mathrm{GHz}$ are discussed.
\end{abstract}

\section{Introduction}

Measurements are needed to evaluate the operation of single gyrotron components itself and further, the assembled gyrotron. This paper summarizes three main measurement facilities for fusion gyrotrons at the KIT/IHM in the year 2018 and possible approaches for upgrading these facilities towards operation above $200 \mathrm{GHz}$ are discussed.

Firstly, the RF window measurement test facility is described which gives the possibility to characterize the RF output window in terms of transmission and reflection features. Secondly, the quasi-optical system consisting of launcher and mirrors can be verified after the fabrication in the low power measurement test facility. Thirdly, the frequency measurement setup is a key instrument for the validation of the behavior of the gyrotron in the high power measurement facility.

\section{Window Measurement Setup}

The RF-window requirements are high temperature conductivity and high RF-power transmission capacity. Chemical vapor deposition (CVD) diamond shows the best properties for this application, so far. The major properties of CVD diamond are: relative permittivity $\varepsilon_{\mathrm{r}}=5.67$, loss tangent $\tan \delta \approx 2 \cdot 10^{-5}$ and thermal conductivity $\kappa \approx 1900 \mathrm{~W} / \mathrm{m} \cdot \mathrm{K}$. The window measurement setup at the KIT/IHM is presented in [1]. The configuration of the setup is a four port scenario. One port is used for transmitting and one for receiving the signal (reflectivity measurement). A beam splitter is required to separate the transmitting (Tx) and receiving $(\mathrm{Rx})$ path. The two unused ports have to be matched perfectly. The matching is realized using absorber material and a ceramic plate which is tilted by the corresponding Brewster angle. The dynamic range of the system is calculated to $60 \mathrm{~dB}$ and the covered frequency range is from $110-170 \mathrm{GHz}$ and is determined by the used frequency multiplier (Tx path) and harmonic mixer (Rx path). The setup can be easily adapted to higher frequencies by changing the above mentioned multiplier and harmonic mixer.

\section{Launcher Measurement Setup}

This setup is used to verify the quasi-optical (q. o.) gyrotron components (launcher and mirrors) at low power $(\sim 1 \mathrm{~mW})$. The availability of such a test system is vital for the gyrotron design because the q. o. output coupler is one of the most critical gyrotron component. This q. o. system converts the rotating cavity mode into a linearly polarized Gaussian beam. Compared with the gyrotron cavity, the low-power test setup is operating without electron beam. Therefore, the correct mode has to be excited in a different way using a mode generator, as depicted in Fig. 1. The design is based on a perforated gyrotron cavity as published in [2].

The RF wave is transmitted using a horn antenna. A satisfying mode is only excited if the wave beam has a minor phase changing. For this reason, a lens system is required to convert the spherical wave into a Gaussian like beam. The quasi-parabolic mirror is necessary to focus the wave on the caustic radius of the preferred mode in the cavity. The cavity is specially designed for this purpose using a scattering matrix code [3]. As in coaxial gyrotrons, an insert is used for mode selection and easier excitation of the mode [4,5]. The midsection of the cavity wall is perforated so that the wave can propagate through the holes.

The excitation of the correct mode is the most challenging task due to the high amount of degrees of freedom of the system. Therefore, the excited mode has to be checked first. For this reason, a non-linear up taper is mounted at the end of the cavity for reaching a better measurement resolution due to the increased diameter. If the mode is excited with sufficient purity, the non-linear up taper will be replaced by the launcher. The excited mode (cavity mode with non-linear up taper or Gaussian beam using the quasi-optical system) is measured with a nearfield pick-up antenna. The antenna is mounted on a 3D measurement arm, where a stepwise scan of the complete plane can be done.

The KIT has developed, in close collaboration with the IPP Garching, mode generators for several operating gyrotron modes ( $\mathrm{TE}_{22,6^{-}}, \mathrm{TE}_{22,8^{-}}, \mathrm{TE}_{28,8^{-}}$,

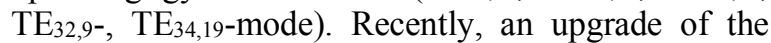
$1 \mathrm{MW}$ hollow-cavity $\mathrm{TE}_{28,8}$-mode gyrotrons operating at Wendelstein 7-X (W7-X) towards 1.5 MW RF output power is under consideration ( $\mathrm{TE}_{28,10}$ mode) [6].

Developments are ongoing targeting more precise measurements and to implement an intelligent 
algorithm achieving a substantial time saving for finding the correct mode in high quality. Further, the RF front end is upgraded to cover the frequency range from $140-330 \mathrm{GHz}$ for future gyrotron components.

\section{Frequency Measurement Setup}

Parts of the gyrotron signal is received by a pickup antenna at the tube's relief window. Then, the signal is divided and feeds two different frequency measurement systems. One of them is called FMS (frequency measurement setup) [7] and the other one is called PMS (pulse spectrum analysis system) [8].

At the beginning of the FMS, the signal is down converted by a harmonic mixer. The mixer operates on the $9^{\text {th }}$ harmonic and has an intermediate frequency (IF) range of $f_{\mathrm{IF}}=0.1-18 \mathrm{GHz}$. The frequency of the IF signal is determined using a filter-bank spectrometer and a frequency time analyzer. The filterbank spectrometer is constructed with multiplexers which divide the $18 \mathrm{GHz}$ spectrum into 9 sub-channels of $\sim 2 \mathrm{GHz}$ bandwidth. Afterwards the signals are detected by wide-band RF diodes and sampled with an oscilloscope. The dynamic range is $24 \mathrm{~dB}$. The filterbank spectrometer gives a broadband but not precise overview about the gyrotron behavior. Therefore, a modulation domain analyzer is implemented in this channel achieving high frequency resolution in a limited frequency bandwidth of around $2 \mathrm{GHz}$. This path offers a dynamic range of $20 \mathrm{~dB}$. The FMS can be easily upgraded by changing the first mixer stage to cover any frequency ranges (e.g. 170$260 \mathrm{GHz}$ ).

The FMS is a very useful setup to get a very quick overview about the behavior of the gyrotron operation. The major drawbacks of the FMS setup are the low dynamic range and the limited bandwidth, where measurements can be performed with high resolution. Therefore, the PSA has been implemented [8]. This system has the following aims: (i) high dynamic range for parasitic oscillation detection, (ii) high frequency resolution for the diagnosis of frequency instabilities and modulation, (iii) high bandwidth for mode competition investigations (mode switching) and (iv) time dependent analysis.

The measurement setup is based on a super-heterodyne receiver. Ambiguities can occur in the RF spectrum reconstruction due to the lack of knowledge if the RF signals are placed in the upper or lower side bands. For this reason, a second identical channel with slightly

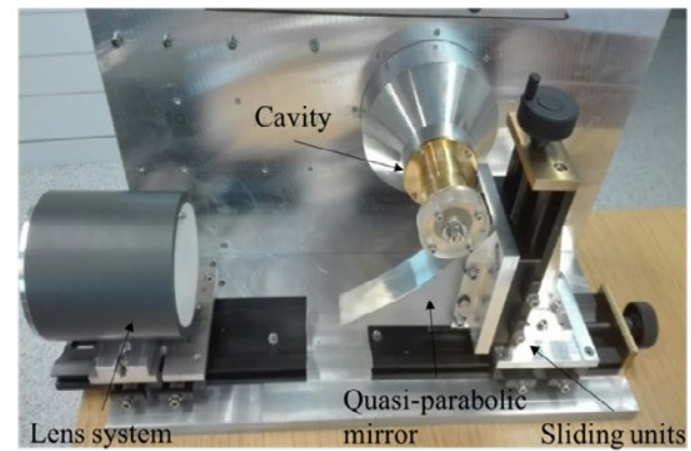

Fig. 1. Mode generator set-up used for the excitation of the correct operating gyrotron cavity mode. different LO (local oscillator) frequency is designed. The effective bandwidth $f_{\mathrm{BW}}$ of the measurement setup can be increased from $f_{\mathrm{BW}}=3-3.5 \mathrm{GHz}$ to $f_{\mathrm{BW}} \approx 6 \mathrm{GHz}$ using the knowledge about the evaluation of both side bands. The effective bandwidth is further increased to $f_{\mathrm{BW}} \approx 12 \mathrm{GHz}$ using a second identical channel with two more mixers. The dynamic range of this setup amounts to $60 \mathrm{~dB}$, which is enough to detect eventual parasitic oscillations.

A possible upgrade is by shunting a second mixer, which operates in a frequency range of from 170$260 \mathrm{GHz}$, as shown in Fig. 2. A diplexer and attenuators can be used to separate the different frequency ranges. Shunting several mixers is a first proposal for upgrading the existing PSA for DEMO relevant gyrotrons operating at frequencies up to $240 \mathrm{GHz}$.

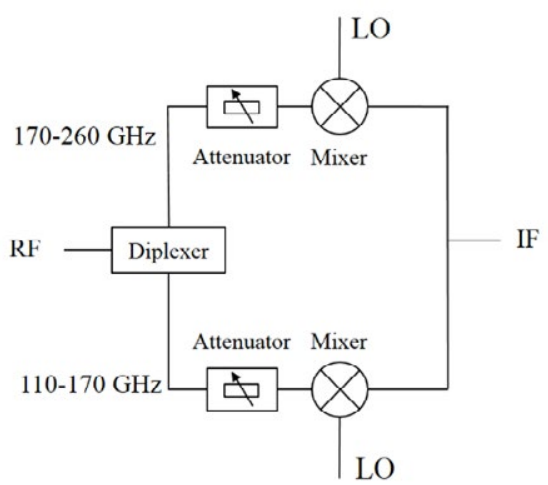

Fig. 2. Possible upgrade of the PSA towards a frequency range from $110-260 \mathrm{GHz}$.

\section{References}

[1]J. Flamm, et al., "Characterization of windows for fusion applications using a D-band network analyzer", $33 \mathrm{rd}$ Int. Conf. on Infrared, Millimeter and Terahertz Waves, Pasadena, CA, 2008, pp. 1-2.

[2] N. L. Alexandrov, et al., "Selective excitation of highorder modes in circular waveguides", Int. J. Infrared and Millimeter Waves, 13, 1369-1385, 1992.

[3] D. Wagner, et al., "Analysis of a complete gyrotron oscillator using the scattering matrix description", Int. J. Infrared and Millimeter Waves, 19, 185-194 (1998).

[4] C. T. Iatrou, S. Kern, A. B. Pavelyev, "Coaxial cavities with corrugated inner conductor for gyrotrons", IEEE Trans. Microwave Theory Tech., 44, pp. 56-64, 1996.

[5] K. A. Avramides, et al., "Design Considerations for powerful continuous-wave second-cyclotron-harmonic coaxial-cavity gyrotrons", IEEE Trans. on Plasma Science, 32, no. 3, 2004.

[6] K. A. Avramides, et al., "Studies towards an upgraded 1.5 MW gyrotron for W7-X", 20th Joint Workshop on Electron Cyclotron Emission (ECE) and Electron Cyclotron Resonance Heating (ECRH), May 2018, Greifswald, Germany.

[7] T. Geist, M. Thumm, W. Wiesbeck, "Contiguous filterbank receiver for a pulsed $140 \mathrm{GHz}$ gyrotron, Conf. Digest, 16th Int. Conf. on Infrared and Millimeter Waves, Lausanne, 1991, Proc., SPIE 1567, pp. 274-275.

[8] A. Schlaich, et al., "Transient millimeter-wave signal analysis with unambiguous RF spectrum reconstruction", IEEE Trans. on Microwave Theory and Techniques, vol. 61, no. 12, pp. 4660-4666, Dec. 2013. 